\title{
Probability Modeling of Precipitation Extremes over Two River Basins in Northwest of China
}

\author{
Zhanling Li, ${ }^{1}$ Zhanjie Li, $^{2}$ Wei Zhao, ${ }^{1}$ and Yuehua Wang ${ }^{1}$ \\ ${ }^{1}$ School of Water Resources and Environment, China University of Geosciences, Beijing 100083, China
}

${ }^{2}$ College of Water Sciences, Beijing Normal University, Beijing 100875, China

Correspondence should be addressed to Zhanling Li; zhanling.li@cugb.edu.cn

Received 1 July 2014; Accepted 3 November 2014

Academic Editor: Francisco J. Tapiador

Copyright (C) 2015 Zhanling Li et al. This is an open access article distributed under the Creative Commons Attribution License, which permits unrestricted use, distribution, and reproduction in any medium, provided the original work is properly cited.

\begin{abstract}
This paper is focused on the probability modeling with a range of distribution models over two inland river basins in China, together with the estimations of return levels on various return periods. Both annual and seasonal maximum precipitations (MP) are investigated based on daily precipitation data at 13 stations from 1960 to 2010 in Heihe River and Shiyang River basins. Results show that GEV, Burr, and Weibull distributions provide the best fit to both annual and seasonal MP. Exponential and Pareto 2 distributions show the worst fit. The estimated return levels for spring MP show decreasing trends from the upper to the middle and then to the lower reaches totally speaking. Summer MP approximates to annual MP both in the quantity and in the spatial distributions. Autumn MP shows a little higher value in the estimated return levels than Spring MP, while keeping consistent with spring MP in the spatial distribution. It is also found that the estimated return levels for annual MP derived from various distributions differ by $22 \%, 36 \%$, and $53 \%$ on average at 20 -year, 50 -year, and 100 -year return periods, respectively.
\end{abstract}

\section{Introduction}

Precipitation extremes, due to the great damage they caused in both social and economic losses, have been caught lots of attention from both governments and the public [1-4]. Understanding of whether and how the frequency and magnitude of precipitation extremes have changed during the past several decades is not only the focus in hydrological, meteorological, climatic, and the related sciences, but also a crucial issue for the management of the associated risks [5-8]. Probability distribution models are the useful tools for the frequency analysis of precipitation extremes [8-10]. However, how to choose an appropriate model in a specific study is still a matter of debate [11].

Many kinds of probability distributions are available to investigate the precipitation extremes. For example, Rahmani et al., [12] used Weibull distribution to calculate the extreme precipitation frequency at stations in Kansas and the adjacent states. Du et al. [8] and Xia et al. [13] used Generalized Extreme Value (GEV) and Generalized Pareto distribution (GPD) to discuss the historical extreme precipitation frequency and its spatio-temporal variations in Haihe and
Huaihe river basins of China. Benyahya et al., [14] compared five probability distributions (GEV, Generalized Logistic, Weibull, Gamma, and Lognormal) to identify the appropriate modes providing the most accurate seasonal maximum precipitation in southern Quebec of Canada. Li et al. [10] discussed the six probability distributions performances (Exponential, Gamma, Weibull, skewed Normal, mixed Exponential, and hybrid Exponential/Generalized Pareto distributions) in precipitation extremes fitting on the Loess Plateau of China based on 47 stations. Rahman et al. [15] investigated the suitability of as many as fifteen different probability distributions based on large Australian annual maximum flood datasets.

Some studies show that GEV or GPD could provide the optimum fit to the extreme precipitation data for comparisons $[6,14,16]$, while others show the different results. Wilks [17] found that the mixed Exponential distribution simulated extremes better than Gamma distribution at 30 stations across the United States. Li et al. [10] concluded that the skewed Normal distribution performed the best in reproducing extreme precipitation events on the Loess Plateau 
of China. Fischer et al. [18] found Wakeby distribution described best for both the maximum precipitation and 5day maximum precipitation in Zhujiang river basin of China. Papalexiou et al. [3] compared the performance of Pareto, Weibull, Lognormal, and Gamma distributions based on daily precipitation records from 15029 stations and found that the ranking from best to worst in terms of their performance was Pareto, Lognormal, Weibull, and Gamma distributions.

Studies also show that the applicability of different probability distributions is found to be related to the spatial and temporal differences in the study area. For example, the mixed Exponential distribution is superior during warmer months, whereas the Gamma distribution is adequate for winter months in Canada [19]. GEV provides a better performance for catchments with medium to high mean annual precipitation, while the 3-parameter Lognormal distribution is probably a more appropriate choice for dry intermediatesized catchments [16]. Additionally, the complexity of probability distributions may contribute to model performance. Suhaila and Jemain [20] assessed the performances of the mixed Gamma, mixed Weibull, and mixed Exponential ones together with single parameter distributions in Peninsular Malaysia and found the mixture distributions to be better than the single distributions in modelling precipitation amounts.

Each kind of probability distribution has its own applicability and limitations. Assessing the applicability of different distribution models is necessary to provide more accurate precipitation information. Furthermore, the selection of an appropriate probability distribution is of prime importance in frequency analysis of extreme events. Consequently, this study will mainly focus on the probability modeling of precipitation extremes over two river basins in China by using different probability distributions, together with the estimations of return periods. This paper aims to contribute to not only providing support in the selection of extreme value techniques for frequency analysis of precipitation extremes over the inland river basins, but also better understanding the extreme information of precipitation over the study areas.

\section{Study Area and Datasets}

The second and the third largest inland river basins, Heihe River basin and Shiyang River basin, in northwest of China are selected as the study areas. Two basins are adjacent regions geographically and located between $37^{\circ}-43^{\circ} \mathrm{N}$ and $97^{\circ}-104^{\circ} \mathrm{W}$. The areas for the basins are $142900 \mathrm{~km}^{2}$ and $41600 \mathrm{~km}^{2}$, respectively. Both two river basins are originated from Qilian Mountain and disappeared in the deserts. The climates are characterized as inlands with cold and dry winters and hot and arid summers. The spatial and temporal distributions of annual mean precipitation are uneven for both basins, decreasing from $300-400 \mathrm{~mm}$ in the southern mountainous area (the upper reaches) to $100 \mathrm{~mm}$ or so in the north (the lower reaches). More than $70 \%$ of annual precipitation concentrates in June, July, August, and September, and that in July is much higher than other months. Winter precipitation (December, January, and February the following year) partly in form of snowfall is the least, only accounting for $3 \%$ or so of the total amount. The coefficient of variation (CV) over Heihe River basin increases from 0.13 to 0.56 from the upper to the lower reaches, indicating that interannual precipitation varies greatly for the lower reaches. Comparatively, it changes smoothly for the whole Shiyang River basin with CV values ranging from 0.19 to 0.28 .

There are 13 national meteorological stations over the two basins, with 4 (TL, YNG, QL, and WSL) in the upper reaches, 6 (SD, ZY, GT, JQ, WW, and YC) in the middle reaches, and 3 (DX, EJNQ, and MQ) in the lower reaches. The elevations range from over $3300 \mathrm{~m}$ to about $900 \mathrm{~m}$. The locations of stations are shown in Table 1 and Figure 1. Daily precipitation records from 13 stations during the period of 1960 to 2010 over two basins are used to assess the performance of a range of probability distribution models.

\section{Methodology}

3.1. Probability Distribution Models. Extreme value theory has emerged as one of the most important statistical disciplines for the meteorological and hydrological sciences over the last 50 years. Extreme value techniques are also becoming widely used in many researches. It is clear that many probability distributions can be used to fit the empirical precipitation extremes $[9,10,15]$. However, we just fit and compare the performance of eight different and commonly used distributions: Exponential, Gumbel, Loglogistic, Lognormal, Pareto 2, Weibull, Burr, and GEV distributions. The reason behind the selection of the above probability distributions is their simplicity, superiority, and popularity in the literatures for frequency analysis of extreme events [11, 15].

The Exponential distribution is a commonly used distribution. It has a fairly simple mathematical form with the probability density function (pdf) and the cumulative density function (cdf) as follows:

$$
\begin{gathered}
f(x)=\lambda \exp (-\lambda x), \\
F(x)=1-\exp (-\lambda x) .
\end{gathered}
$$

It requires the knowledge of only one parameter, $\lambda$, which makes it fairly easy to manipulate. As $\lambda$ is decreased, the distribution is stretched out to the right, and as $\lambda$ is increased, the distribution is pushed towards the origin. It is a special case of the Weibull distribution.

The Gumbel distribution also referred to as the extreme value type I distribution [9] has two forms, one is based on the smallest extreme (minimum case), and the other is based on the largest extreme (maximum case). In this study, the maximum case is used. Its pdf and cdf are given by

$$
\begin{gathered}
f(x)=\frac{1}{\sigma} \exp \left[-\left(\frac{x-\mu}{\sigma}\right)-\exp \left(-\frac{x-\mu}{\sigma}\right)\right], \\
F(x)=\exp \left[-\exp \left(-\frac{x-\mu}{\sigma}\right)\right]
\end{gathered}
$$

and $\sigma$ is scale parameter $(\sigma>0)$ and $\mu$ is location parameter. It is also a particular case of GEV distribution. 


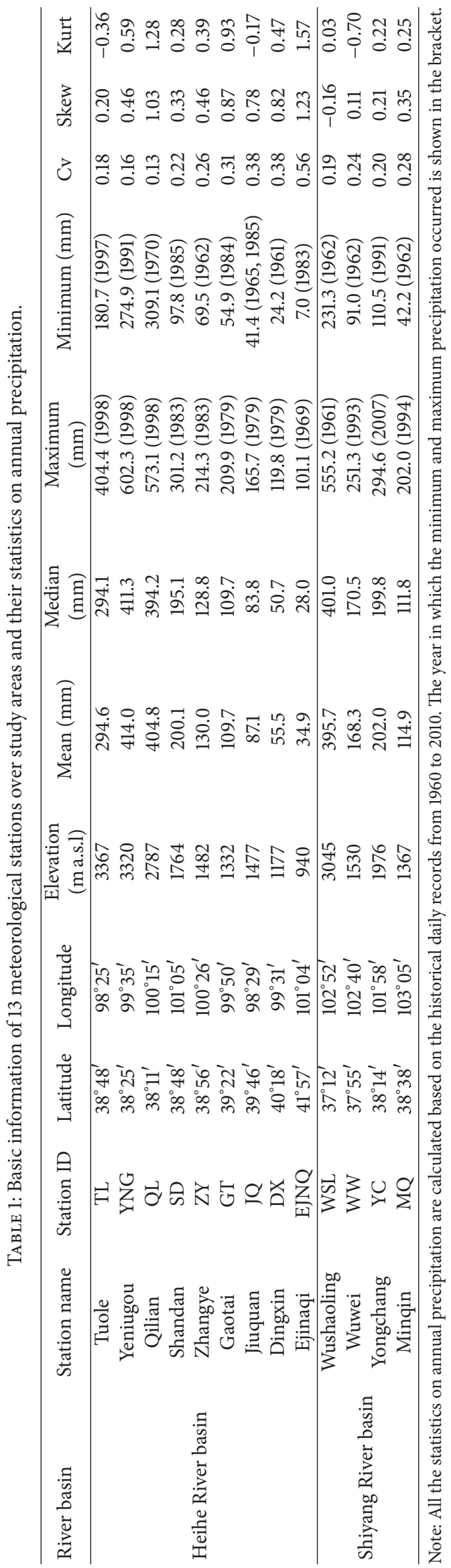




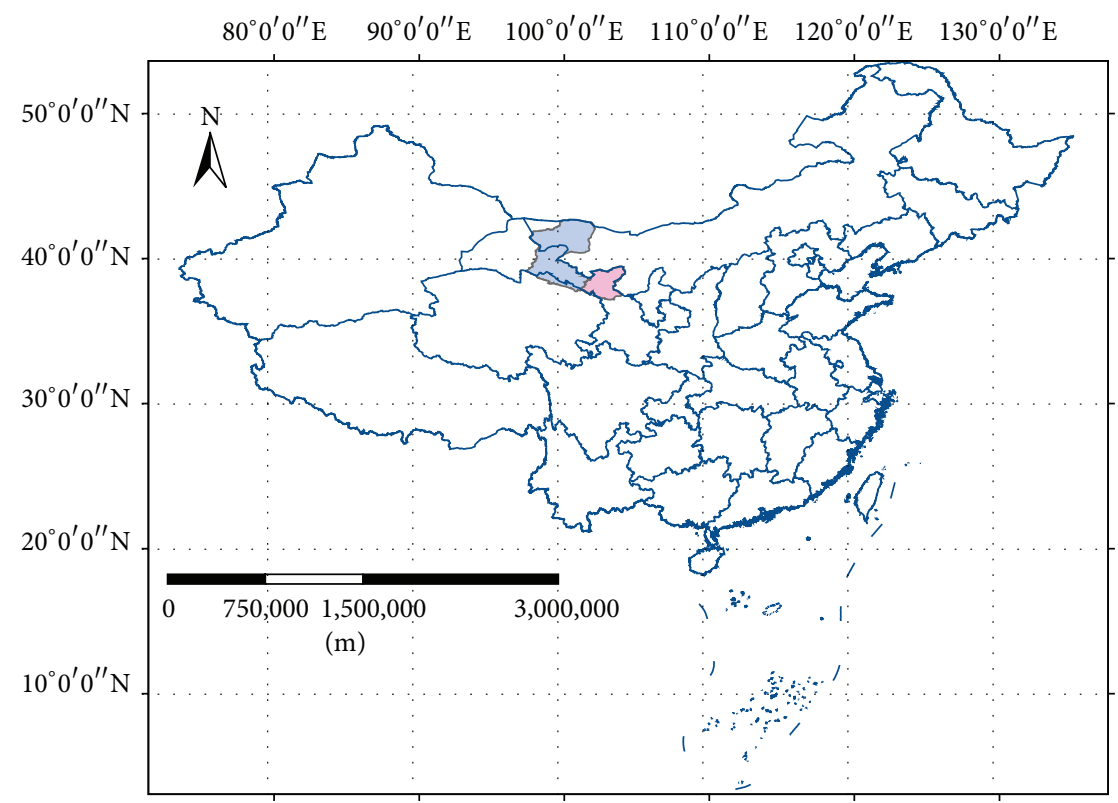

Heihe River basin

Shiyang River basin

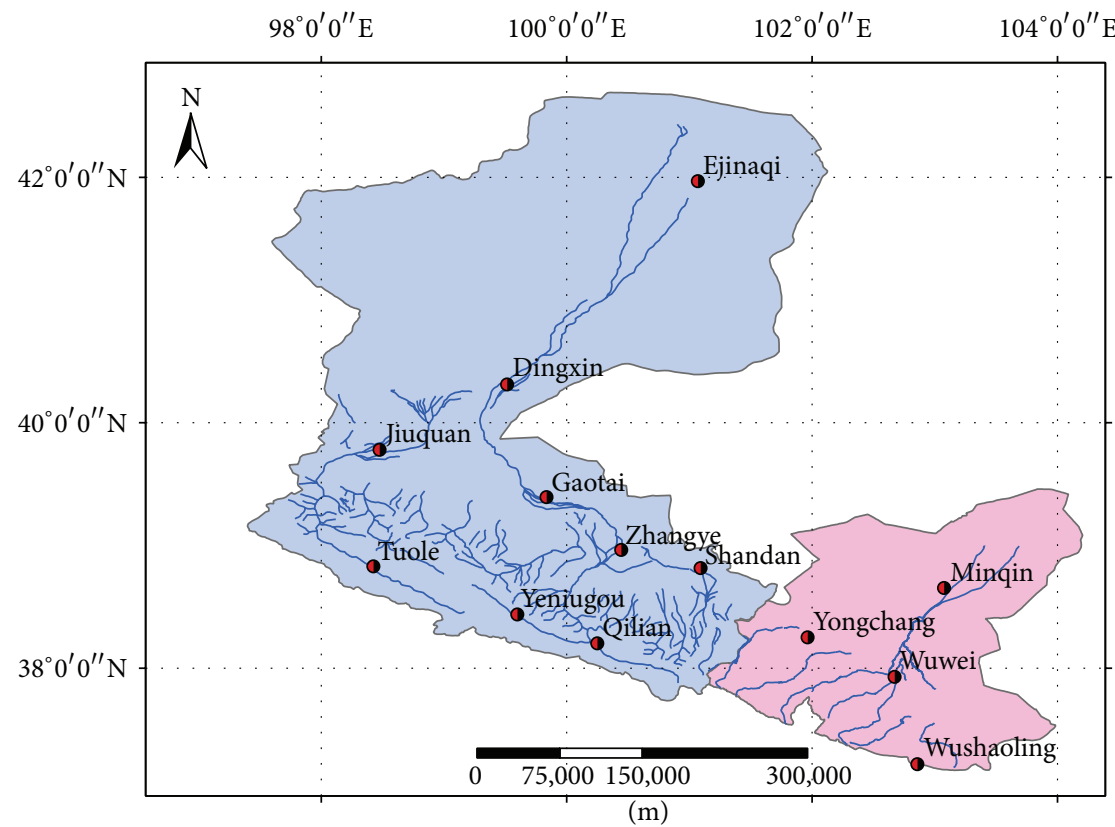

- Meteorological station

River

Heihe River basin

Shiyang River basin

FIGURE 1: The locations of Heihe River basin and Shiyang River basin in China and the meteorological stations involved.

The Loglogistic distribution is a two-parameter distribution with parameters $\alpha$ and $\beta$. Its pdf and cdf are given by

$$
F(x)=\left[1+\left(\frac{x}{\alpha}\right)^{-\beta}\right]^{-1}
$$

$$
f(x)=\frac{(\beta / \alpha)(x / \alpha)^{\beta-1}}{\left[1+(x / \alpha)^{\beta}\right]^{2}},
$$

and $\alpha$ is shape parameter $(\alpha>0)$ and $\beta$ is scale parameter $(\beta>0)$. For $\beta \geq 1$, its pdf decreases monotonically and is 
convex. For $0<\beta<1$, the pdf starts at zero, increases to its mode, and decreases thereafter. The shape of the Loglogistic distribution is very similar to that of the Lognormal distribution and the Weibull distribution.

The Lognormal distribution, as may be surmised by the name, has certain similarities to the Normal distribution. A random variable is Lognormally distributed if the logarithm of the random variable is normally distributed. The pdf and cdf for Lognormal distribution are given by

$$
\begin{gathered}
f(x)=\frac{1}{x \sigma \sqrt{2 \pi}} \exp \left[-\frac{(\ln x-\mu)^{2}}{2 \sigma^{2}}\right], \\
F(x)=\Phi\left(\frac{\ln x-\mu}{\sigma}\right),
\end{gathered}
$$

where $\Phi$ is the Laplace Integral, $\sigma$ is scale parameter $(\sigma>0)$, and $\mu$ is location parameter. The Lognormal distribution is a good companion to the Weibull distribution in many cases.

The Pareto distribution is a power law probability distribution that is used in description of many types of observations. There is a hierarchy of Pareto distributions known as Pareto types I, II, III, and IV and Feller-Pareto distributions. The pdf and cdf for Pareto type II (written as Pareto 2 herein) which is employed in this study are given by

$$
\begin{gathered}
f(x)=\frac{\alpha \beta^{\alpha}}{(x+\beta)^{\alpha+1}}, \\
F(x)=1-\left(\frac{\beta}{x+\beta}\right)^{\alpha}
\end{gathered}
$$

and $\alpha$ is shape parameter $(\alpha>0)$ and $\beta$ is scale parameter $(\beta>0)$. The Pareto distribution is a special case of the GPD which is a widely used and popular extreme value technique in recent years $[1,10,13,21]$.

The Weibull distribution, also known as extreme value type III distribution, is still a two-parameter distribution with parameters $\alpha$ and $\beta$. The $\mathrm{pdf}$ and cdf for Weibull distribution are

$$
\begin{gathered}
f(x)=\frac{\alpha}{\beta}\left(\frac{x}{\beta}\right)^{\alpha-1} \exp \left[-\left(\frac{x}{\beta}\right)^{\alpha}\right], \\
F(x)=1-\exp \left[-\left(\frac{x}{\beta}\right)^{\alpha}\right]
\end{gathered}
$$

and $\alpha$ is shape parameter $(\alpha>0)$ and $\beta$ is scale parameter $(\beta>0)$. The Weibull distribution is a versatile distribution that can take on the characteristics of other types of distributions, based on the value of the shape parameter, $\beta$.

The three-parameter Burr distribution is a very flexible distribution family that can express a wide range of distribution shapes. The pdf and cdf for Burr distribution are given by

$$
\begin{gathered}
f(x)=\frac{\alpha k(x / \beta)^{\alpha-1}}{\beta\left[1+(x / \beta)^{\alpha}\right]^{k+1}}, \\
F(x)=1-\left[1+\left(\frac{x}{\beta}\right)^{\alpha}\right]^{-k},
\end{gathered}
$$

where $k$ is shape parameter $(k>0), \alpha$ is shape parameter $(\alpha>$ $0)$, and $\beta$ is scale parameter $(\beta>0)$. The Burr distribution includes, overlaps, or has as a limiting case many commonly used distributions such as Gamma, Lognormal and Loglogistic ones. It has two asymptotic limiting cases: Weibull and Pareto type I. Due to different values of its parameters covering a broad set of skewness and kurtosis, the Burr distribution can fit a wide range of empirical data in various fields such as hydrology, meteorology, and finance.

The generalized extreme value (GEV) distribution is a family of continuous probability distributions developed within extreme value theory to combine the Gumbel, Fréchet, and Weibull families [9]. By the extreme value theorem, it is the limiting distribution of an infinite observed series of maximum (minimum) values that are independent and identically distributed. The pdf and cdf for GEV are given by

$$
\begin{gathered}
f(x)=\frac{1}{\sigma}\left[\left(1+\xi \frac{x-\mu}{\sigma}\right)^{-1 / \xi}\right]^{\xi+1} e^{-\left[(1+\xi((x-\mu) / \sigma))^{-1 / \xi}\right]} \quad \xi \neq 0 \\
F(x)=e^{-[1+\xi((x-\mu) / \sigma)]^{-1 / \xi}} \quad \xi \neq 0
\end{gathered}
$$

where $x$ is a selected quantile and $\mu, \sigma$, and $\xi$ are referred to as the location, scale, and shape parameters, respectively. When the shape parameter $\xi>0$, the distribution is said to have a heavy tail and is called GEV type II or Frechet distribution. In this case, the probability density function decreases in the upper tail at a very slow rate. When $\xi<0$ then the distribution has a bounded (from below and above) upper tail (GEV type III or the Weibull distribution). The $\xi=0$ is a special case of the GEV distribution (GEV type I) that is referred to as the Gumbel distribution.

It is well known that several methods have been extensively used to estimate the parameters of candidate probability distributions, for example, the maximum likelihood method, method of moments, method of L-moments, and least square method [22-24]. We employ the widely used maximum likelihood estimation to derive the parameters for different probability distributions.

3.2. Goodness-of-Fit. Kolmogorov-Smirnov test is used in this study to decide if the precipitation extremes come from a hypothesized continuous distribution. It is based on the empirical cumulative distribution function (cdf). Assume that we have a random sample $x_{1}, \ldots, x_{n}$ from some distribution with $\operatorname{cdf} F(x)$. The empirical cdf is denoted by

$$
F_{n}(x)=\frac{1}{n}[\text { number of observations } \leq x] .
$$

The Kolmogorov-Smirnov statistic $(D)$ is based on the largest vertical difference between the theoretical cdf and the empirical cdf

$$
D=\max _{1 \leq i \leq n}\left[F\left(x_{i}\right)-\frac{i-1}{n}, \frac{i}{n}-F\left(x_{i}\right)\right] .
$$

The hypothesis regarding the distributional form is rejected (the data do not follow the specified distribution) at the 
chosen significance level $\alpha$ if the test statistic, $D$, is greater than the critical value obtained from a table [25]. The fixed values of $\alpha(0.01,0.05$, etc.) are generally used to evaluate the null hypothesis $\left(H_{0}\right)$ at various significance levels. A value of 0.05 is typically used for most applications and also for this study.

Additionally, the probability difference graph and the probability-probability (P-P) plot are used as well to evaluate the quality of the model fitting. The probability difference graph is a plot of the difference between the empirical cdf and the theoretical cdf,

$$
\operatorname{Diff}(x)=F_{n}(x)-F(x) .
$$

This graph can be used to determine how well the theoretical distribution fits to the observed data and compare the goodness of fit of several fitted distributions. The P-P plot is a graph of the empirical cdf values plotted against the theoretical cdf values. It is used to determine how well a specific distribution fits to the observed data. This plot will be approximately linear if the specified theoretical distribution is the correct model [9].

3.3. Return Level Estimation. When we explore the extreme events, what extreme events might occur in 100-year or even longer return periods given the much shorter period data available is another active topic $[8,26-28]$. The T-year return level can be calculated straightforwardly once the model parameters are estimated. For example, let $\widehat{\mu}, \widehat{\sigma}$, and $\widehat{\xi}$ be the maximum likelihood estimate for the location $(\mu)$, scale $(\sigma)$, and shape $(\xi)$ parameters of GEV distribution; according to the formula (9), we can get the following equation:

$$
x=\widehat{\mu}-\frac{\widehat{\sigma}}{\widehat{\xi}}\left[1-(-\ln F(x))^{-\widehat{\xi}}\right]
$$

with $F(x)=1-1 / T$; that is,

$$
x=\widehat{\mu}-\frac{\widehat{\sigma}}{\widehat{\xi}}\left[1-\left(-\ln \left(1-\frac{1}{T}\right)\right)^{-\widehat{\xi}}\right]
$$

in which $T$ means the return period and $x$ denotes the theoretical return levels for a given return period. The calculations for other models are the same as this example. The empirical return period for precipitation extremes is calculated with $T=1 /(1-(i-0.4) /(n+0.2))$, in which $i$ means the order of the ascending precipitation extremes data $(i=1,2, \ldots, n)$ [29].

\section{Results and Analysis}

We focus on the precipitation extremes over the two inland river basins, while, what extremes can be considered as "precipitation extremes"? From meteorological point of view, the most commonly used way to indicate the so-called precipitation extremes is those that occurred with the amount exceeding a certain fixed level. However, such fixed level seems not reasonable, since it may vary with the regions and seasons. Thus in some literatures, another way, precipitation that

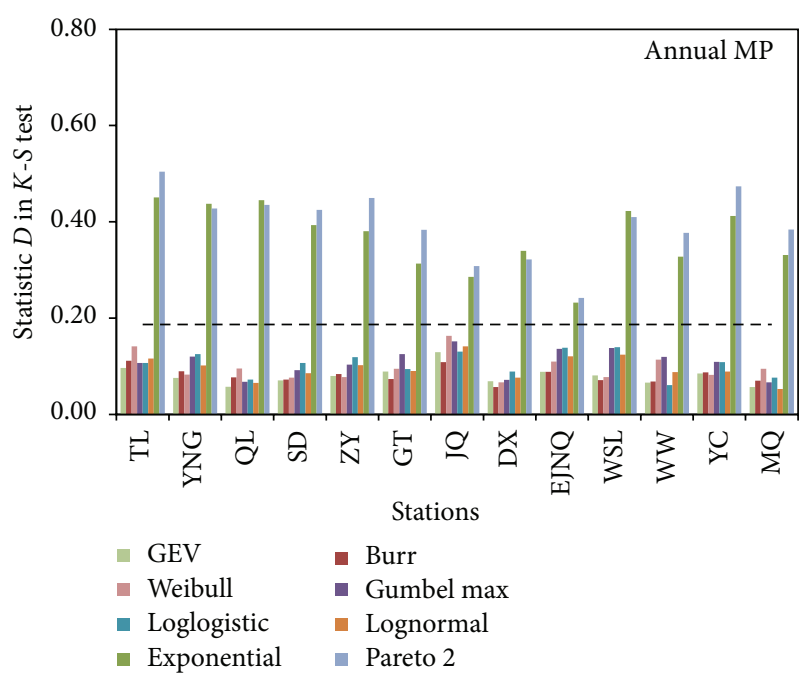

FIgURE 2: Values of statistic D in K-S test for annual maximum precipitation of all stations by using different distributions.

exceed a certain threshold is regarded as precipitation extremes $[9,13,30]$. The advantage of this way lies in making full use of the extreme information contained in a given period, while the limitation is that it is difficult to select the suitable threshold value which requires neither too low nor too high $[6,31]$. Therefore, the series of annual maximum of contiguous daily precipitation observations will be considered as "precipitation extremes" herein. In addition, for better understanding the characteristics of seasonal precipitation extremes, the seasonal maximum precipitation is also investigated in the following analysis.

4.1. Probability Modeling for Annual Maximum Precipitation. Figure 2 shows the values of statistic, $D$, in K-S test for all stations by using the eight distribution models. The smaller the value of statistic $D$, the better the performance of model fitting. The black dashed horizontal line means the critical value at 0.05 significance level. Clearly to see, the hypothesis regarding the annual maximum precipitation (MP) data following the Exponential or Pareto 2 distributions is rejected for all stations at 0.05 significance level since the calculated $D$ is much greater than the critical value. We rank the distributions based on their performances measured by K-S test results. The top three distributions (with the smallest, the second smallest, and the third smallest values of $D$ ) for different stations are shown in Table 2. From Figure 2 and Table 2, it is clear that GEV distribution shows the most appropriate distribution for 5 of 13 annual MP time series, owing to the smallest calculated statistics $D$ among the eight distributions. For another four annual MPs (GT, JQ, DX, and WSL), Burr distribution shows the best partly owing to its flexibility. For ZY and YC stations, Weibull performs the best. Integrating the performance of top three distributions together, GEV ranks the first, owing to 13 annual MP datasets fitted well with the best, the second best, or the third best performance. Burr ranks the second with 10 of 13 datasets fitted well with top three, and Weibull distribution ranks the third with 7 of 13 


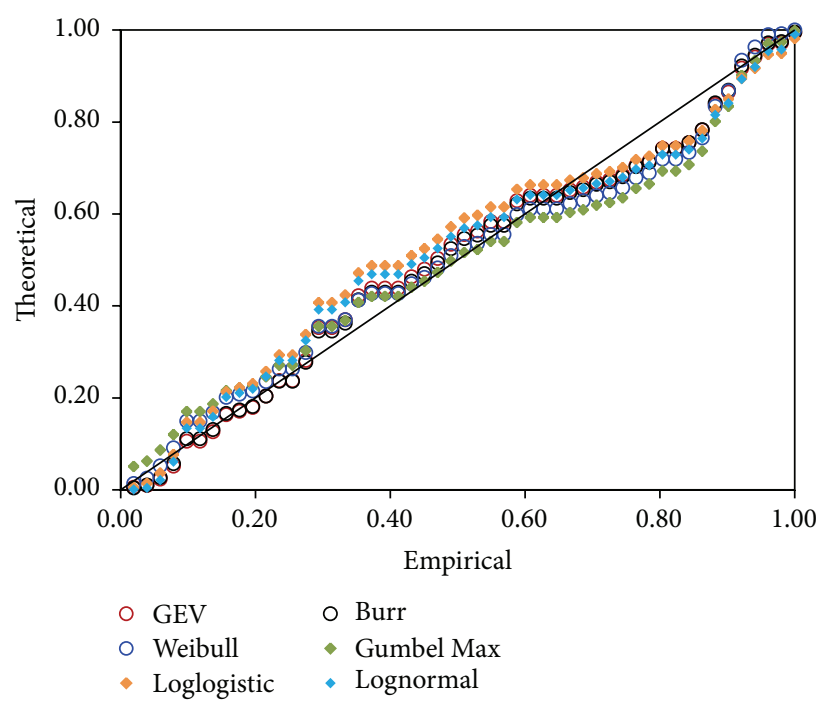

(a)

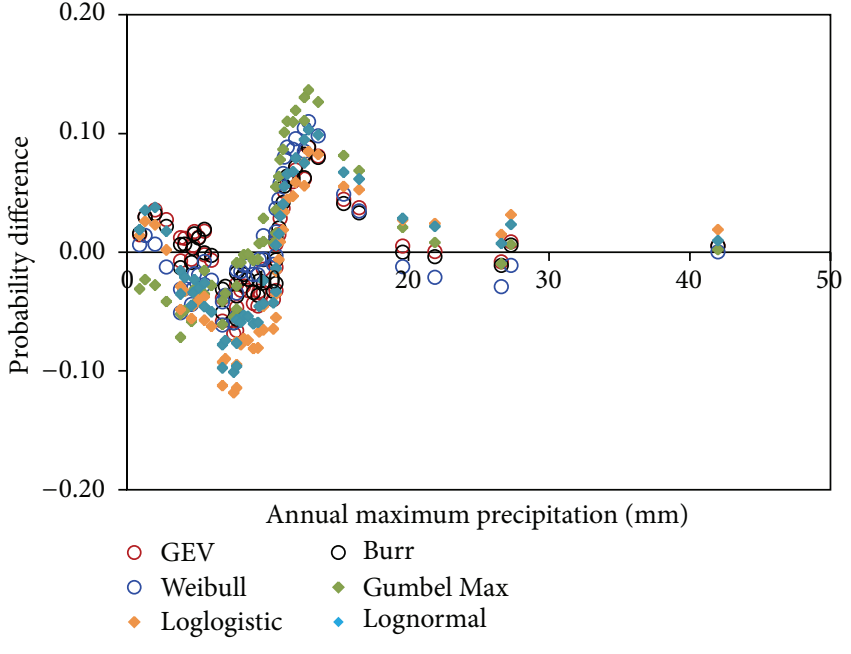

(b)

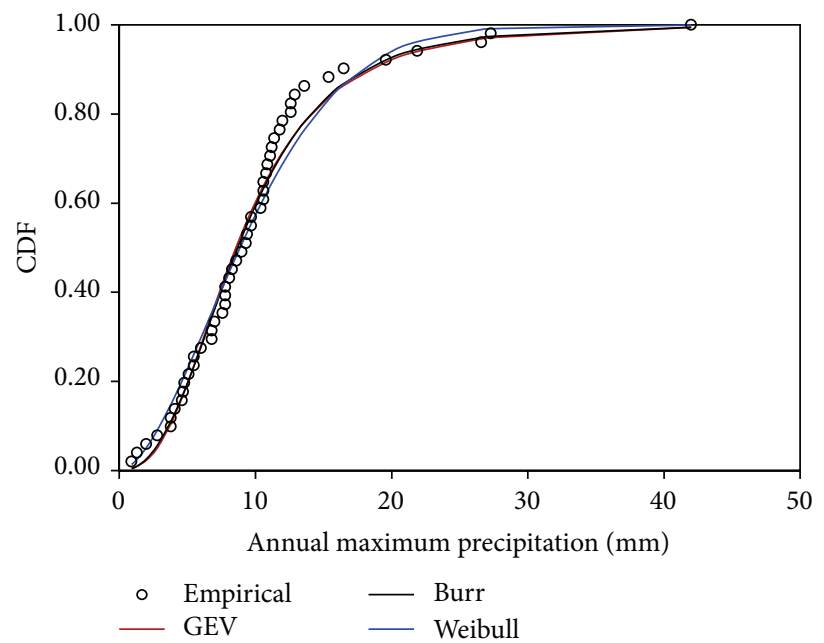

(c)

Figure 3: The P-P plot (a), probability difference graph (b), and cdf plot (c) for annual maximum precipitation of EJNQ station by using various probability distributions.

datasets fitted well with top three. Therefore, GEV, Burr, and Weibull are the top three distributions for the annual MP of this study.

Graphical diagnostics are also explored for evaluating the quality of the model fitting. In a P-P plot, a perfect fit is indicated if all scatters follow the 1:1 line in the plot. The larger the differences between the dots and the line, the poorer the quality of the model fitting. Taking EJNQ station as an example, results for different distributions with better performance in the annual MP fitting are presented for comparisons, which is shown in Figure 3. In the P$\mathrm{P}$ plot (Figure 3(a)), compared with those of other three distributions (Loglogistic, Lognormal, and Gumbel denoted by green, orange, and blue solid rectangles), results for GEV, Burr, and Weibull (denoted by red, black, and blue hollow circles) distributions are more close to the 1:1 line, meaning that the latter specified theoretical distributions are the more appropriate models for comparisons. The same conclusion also can be drawn from Figure 3(b); the smaller probability differences are found for GEV, Burr, and Weibull distributions compared with the other three distributions. Figure 3(c) presents the cdf plot of top three distributions, together with the empirical ones marked by black hollow circles. It is clear that the lines for GEV, Burr, and Weibull models are very close to each other and approximate to most of the empirical points. Graphical diagnostics for other stations also show that satisfactory fittings could be given from the six distributions for each station and fairly better fittings are given from those top three distributions. Due to the space limited, the figures of P-P plots, probability difference plots, and cdf plots for other stations are omitted herein.

4.2. Probability Modeling for Seasonal Maximum Precipitation. With regard to the seasonal MP, four seasons are 


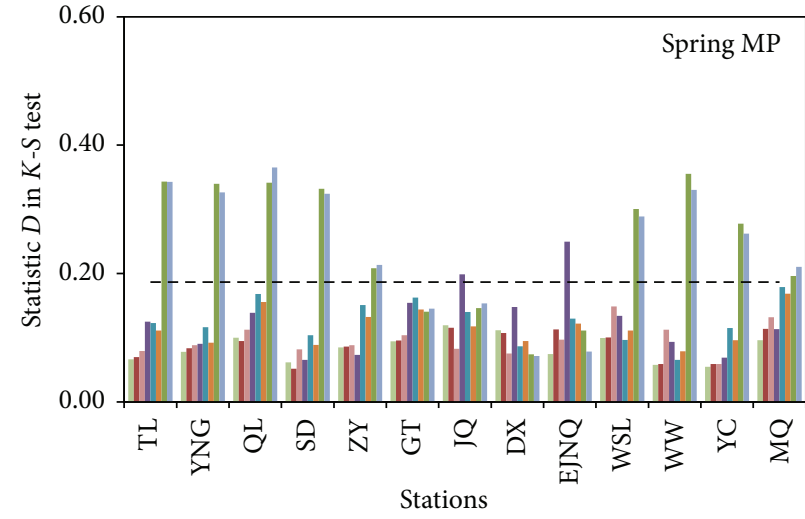

(a)

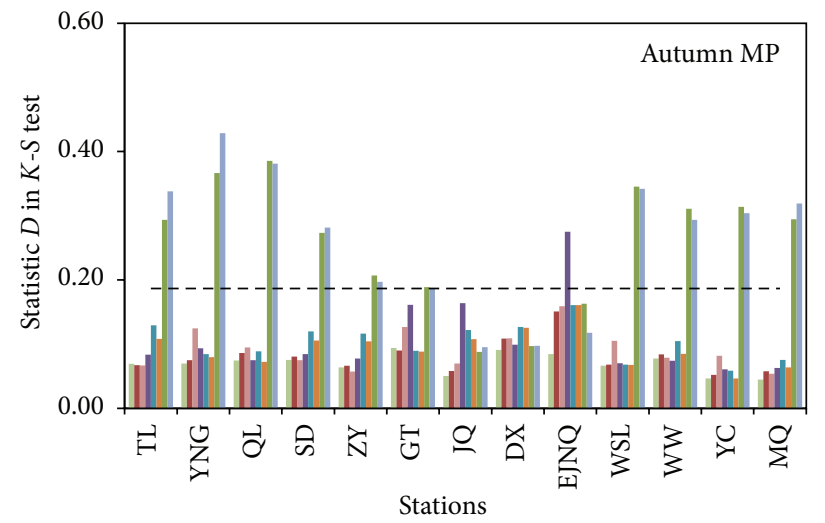

(c)

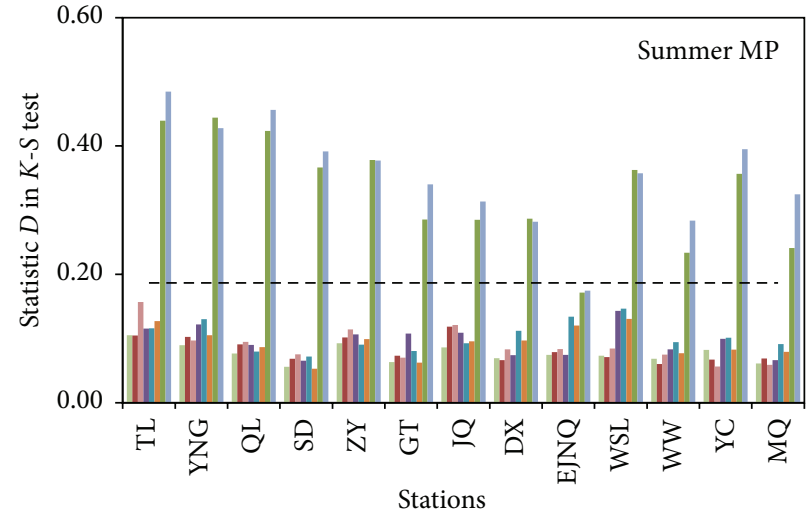

(b)

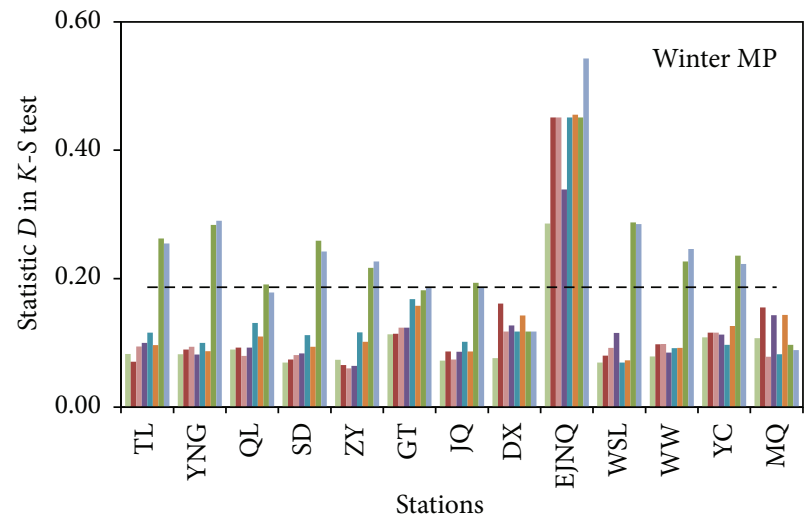

(d)

FIGURE 4: Values of statistic $D$ in K-S test for seasonal maximum precipitation of all stations by using different distributions.

TABLE 2: The top three distributions most suitable for annual maximum precipitation of each station.

\begin{tabular}{lccc}
\hline Station ID & 1 & 2 & 3 \\
\hline TL & GEV & Loglogistic & Gumbel \\
YNG & GEV & Weibull & Burr \\
QL & GEV & Lognormal & Gumbel \\
SD & GEV & Burr & Weibull \\
ZY & Weibull & GEV & Burr \\
GT & Burr & GEV & Lognormal \\
JQ & Burr & GEV & Loglogistic \\
DX & Burr & Weibull & GEV \\
EJNQ & GEV & Burr & Weibull \\
WSL & Burr & Weibull & GEV \\
WW & Loglogistic & GEV & Burr \\
YC & Weibull & GEV & Burr \\
MQ & Lognormal & GEV & Gumbel \\
\hline
\end{tabular}

divided in one year, Spring (March to May), Summer (June to August), Autumn (September to November), and Winter (December to the next February). The MP data over each season then forms the seasonal MP datasets. The eight distributions mentioned above are employed to fit the seasonal maximum datasets as well. Results of K-S test are shown in Figure 4. The legends are the same as those in Figure 2 and thus omitted. As annual ones, Exponential and Pareto 2 models are not proved to be suitable for most of stations (Figure 4) due to the larger values of statistic $D$ in K-S test compared with the critical value denoted by dashed line in the figure.

The top three distributions for each station are shown in Table 3. For Spring MP, GEV performs the best fit for 7 of 13 stations. Burr performs the best for 2 stations. Weibull, Gumble, and Loglogistic ones show the best for only 1 station, respectively. GEV, Burr, and Weibull rank the top three for all datasets, in which 11, 11, and 8 of 13 datasets are fitted well with the best, the second, or the third best performance, respectively. Note that although Pareto 2 and Exponential distributions are not suitable for most datasets, they show the best and the second best performance for Spring MP of DX station and Pareto 2 show the second best for EJNQ station. For Summer MP, the top three distributions for each station are more various. While GEV, Burr, and Weibull still rank the top three for all Summer MP datasets with the best (or second and third best) performance of fitting. Concerning Autumn MP, GEV presents the best fitting for 7 of 13 stations, followed by Weibull and Lognormal distributions. The top three distributions for Autumn MP remain the same as those for other 


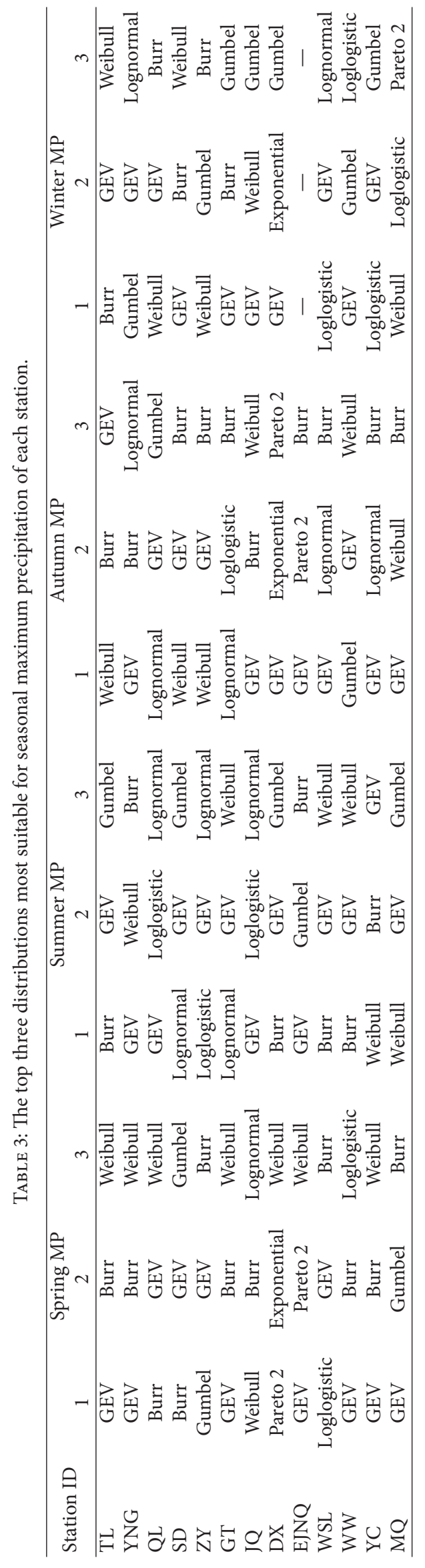


TABLE 4: Estimations of annual and seasonal maximum precipitation at different return periods by using the best fitted model (mm).

\begin{tabular}{|c|c|c|c|c|c|c|c|c|c|c|c|c|c|c|c|}
\hline \multirow{2}{*}{ Station ID } & \multicolumn{3}{|c|}{ Annual } & \multicolumn{3}{|c|}{ Spring } & \multicolumn{3}{|c|}{ Summer } & \multicolumn{3}{|c|}{ Autumn } & \multicolumn{3}{|c|}{ Winter } \\
\hline & (1) & (2) & (3) & (1) & (2) & (3) & (1) & (2) & (3) & (1) & (2) & (3) & (1) & (2) & (3) \\
\hline$\overline{T L}$ & 34 & 39 & 42 & 18 & 19 & 20 & 32 & 45 & 53 & 19 & 21 & 22 & 3 & 4 & $\overline{5}$ \\
\hline YNG & 37 & 40 & 42 & 19 & 22 & 24 & 37 & 40 & 43 & 27 & 33 & 37 & 3 & 4 & 4 \\
\hline QL & 38 & 43 & 46 & 19 & 21 & 22 & 38 & 43 & 46 & 26 & 31 & 34 & 3 & 3 & 3 \\
\hline SD & 41 & 47 & 51 & 18 & 21 & 25 & 43 & 51 & 57 & 23 & 26 & 28 & 4 & 5 & 5 \\
\hline ZY & 28 & 30 & 32 & 17 & 21 & 24 & 30 & 38 & 45 & 20 & 23 & 26 & 3 & 4 & 4 \\
\hline GT & 35 & 45 & 54 & 22 & 30 & 37 & 32 & 39 & 45 & 24 & 33 & 40 & 4 & 5 & 6 \\
\hline JQ & 36 & 47 & 57 & 16 & 20 & 22 & 30 & 39 & 47 & 20 & 29 & 39 & 4 & 5 & 6 \\
\hline DX & 21 & 25 & 29 & 14 & 19 & 22 & 20 & 25 & 29 & 16 & 21 & 25 & 4 & 5 & 7 \\
\hline EJNQ & 23 & 30 & 36 & 10 & 16 & 23 & 18 & 22 & 25 & 14 & 23 & 33 & - & - & - \\
\hline WSL & 38 & 41 & 42 & 26 & 35 & 44 & 37 & 40 & 42 & 28 & 34 & 39 & 4 & 5 & 6 \\
\hline WW & 42 & 55 & 66 & 17 & 21 & 24 & 44 & 57 & 69 & 23 & 28 & 31 & 5 & 6 & 6 \\
\hline YC & 32 & 34 & 36 & 17 & 19 & 21 & 32 & 35 & 37 & 24 & 29 & 33 & 4 & 5 & 7 \\
\hline MQ & 38 & 45 & 50 & 15 & 18 & 20 & 36 & 42 & 45 & 18 & 21 & 23 & 4 & 5 & 6 \\
\hline
\end{tabular}

Note: (1), (2), and (3) mean 20-year, 50-year, and 100-year return periods, respectively.

seasons. Exponential and Pareto 2 models are not suitable for 10 of 13 stations in terms of K-S test. For Winter MP, GEV shows the best fitting for 5 stations, and Weibull and Loglogistic models show the best fitting for 3 and 2 stations. The top three distributions for Winter MP are still GEV, Burr, and Weibull in turn. There is one point that is noteworthy: none of the distributions employed in this study are suitable for Winter MP of EJNQ station. Further analysis for Winter MP of EJNQ station found that the average Winter MP only amounts to $0.43 \mathrm{~mm}$ over the period of 1960 to 2010 and $45 \%$ ( 23 of 51 ) of zero values involved. Therefore, there is not necessary to analyze the Winter MP of this station owing to too small amount of precipitation.

Another comparison revealing the overall performance of the fitting is based on the graphical diagnostics. By way of example, cdf plot for DX station is considered in Figure 5 showing the empirical cdf and the most appropriate theoretical cdf against observed seasonal MP. The hollow circles mean the empirical values and the solid lines mean the theoretical values. The red, black, blue, and green represent Spring, Summer, Autumn, and Winter, respectively. It is clear that the corresponding theoretical distributions fit well not only to the small and moderate points but also to the large (also called the tails) points of each dataset, ensuring that the fitted distribution provides the best possible description of all datasets in four seasons. The cdf plots of seasonal MP for other stations (omitted here due to the large number of figures and space limited) also show that the top one distribution could give a quite satisfactory fitting to the empirical one.

To sum up, GEV, Burr, and Weibull distributions are proved to be most suitable extreme value techniques for annual and seasonal MP over the two river basins.

4.3. Return Level Estimation with the Best Fitted Model. The estimated return levels based on the corresponding optimum models for annual and seasonal MP are given in Table 4. For annual MP, it is estimated to be $37 \mathrm{~mm}$ for the upper reaches on average, $36 \mathrm{~mm}$ for the middle and $27 \mathrm{~mm}$ for the lower

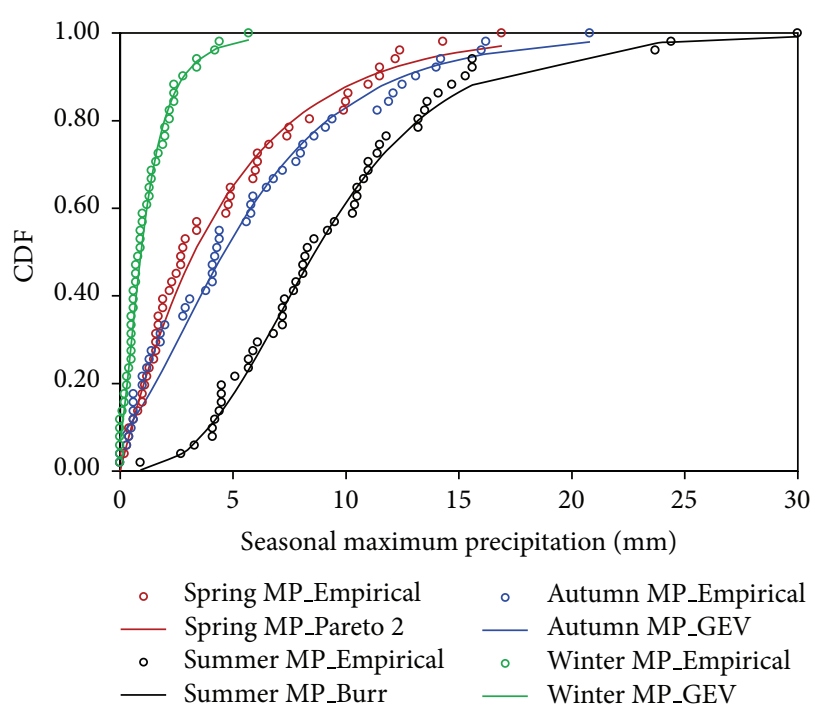

FIGURE 5: The cumulative density function plot for seasonal maximum precipitation of DX station by using the best fitted probability distributions.

reaches at 20-year return period, $41 \mathrm{~mm}, 43 \mathrm{~mm}$, and $33 \mathrm{~mm}$ at 50 -year return period, and $43 \mathrm{~mm}, 49 \mathrm{~mm}$, and $38 \mathrm{~mm}$ at 100 -year return period, showing that, at shorter return period, the estimated return levels are higher for both upper and middle reaches than that for the lower reaches, which is consistent with the spatial changes of annual precipitation. While being at moderate and longer return periods, the situation appears different. The estimated annual MP for the middle reaches shows the highest, followed by those for the upper and lower reaches. Actually, it can be interpreted based on the observed historical precipitation data. For example, the MP for GT station (located in the middle reaches of Heihe River basin) accounts for $65.5 \mathrm{~mm}$ in 1974, more than 1.5 times higher than that for the upper reaches in Heihe River basin (44.2 $\mathrm{mm}$ in 1983 for TL station); the MP of $62.7 \mathrm{~mm}$ 


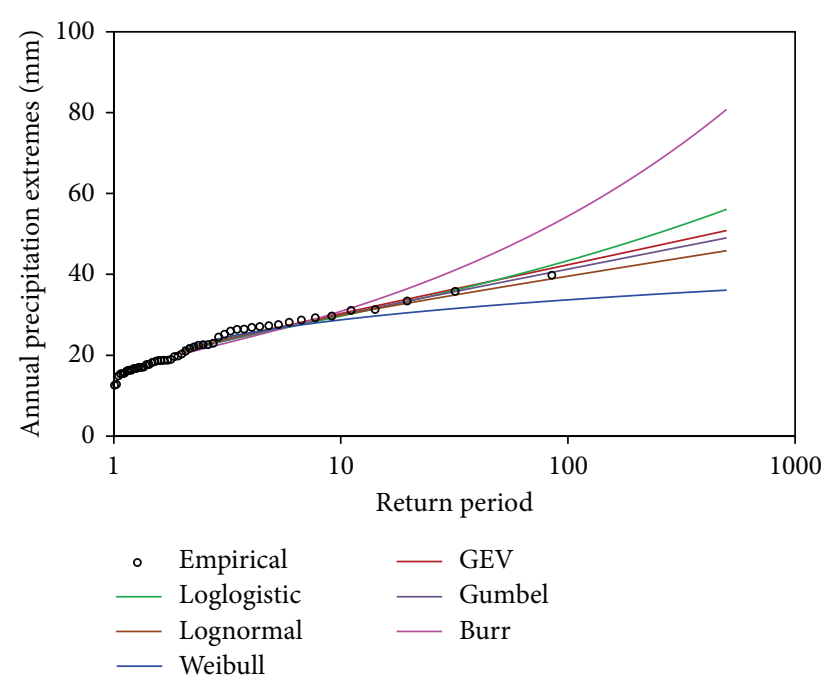

Figure 6: The empirical and the theoretical return levels in terms of different probability distributions for annual maximum precipitation of TL station.

and $65.4 \mathrm{~mm}$ for WW and YC stations observed in 1985 and 1987 is also 1.5 times higher than that for the upper reaches in Shiyang River basin (42.6 mm in 1997 for WSL station). In extreme value theory, the larger values (the tails of the data) are paid more attention in the fitting; thus, the higher historical extreme observations for the middle reaches would largely affect the shape of the distributions and consequently result in larger estimated return levels than those for the upper and lower reaches.

The return levels for Spring MP show decreasing trends from the upper to the middle and then to the lower reaches totally speaking. Specially, it decreases from $21 \mathrm{~mm}$ to $18 \mathrm{~mm}$ and $13 \mathrm{~mm}$ at 20 -year return period, from $24 \mathrm{~mm}$ to $22 \mathrm{~mm}$ and $18 \mathrm{~mm}$ at 50 -year return period, and from $28 \mathrm{~mm}$ to $26 \mathrm{~mm}$ and $22 \mathrm{~mm}$ at 100 -year return period. Summer MP approximates to annual MP both in the estimated quantity and in the spatial distributions, showing higher estimated values for the upper and middle reaches at shorter return period and highest estimated values for the middle reaches at moderate and longer return periods. Estimated values for Autumn MP show a little higher than those for Spring MP, while keeping consistent with those for Spring MP in the spatial distribution. As for Winter MP, very slight precipitation is estimated on three kinds of return periods owing to the dry winter in the study areas.

4.4. Differences in Return Level Estimation. Since more than one distribution model reveals better performance in annual and seasonal MP fittings based on K-S test, we then calculate and compare the estimated return levels in terms of different distributions on various return periods. Taking annual MP of TL station as an example, Figure 6 shows the theoretical return levels in terms of different probability distributions, in which, the red, green, purple, brown, pink, and blue solid lines denote the estimations from GEV, Loglogistic, Gumbel, Lognormal, Burr and Weibull distributions, and

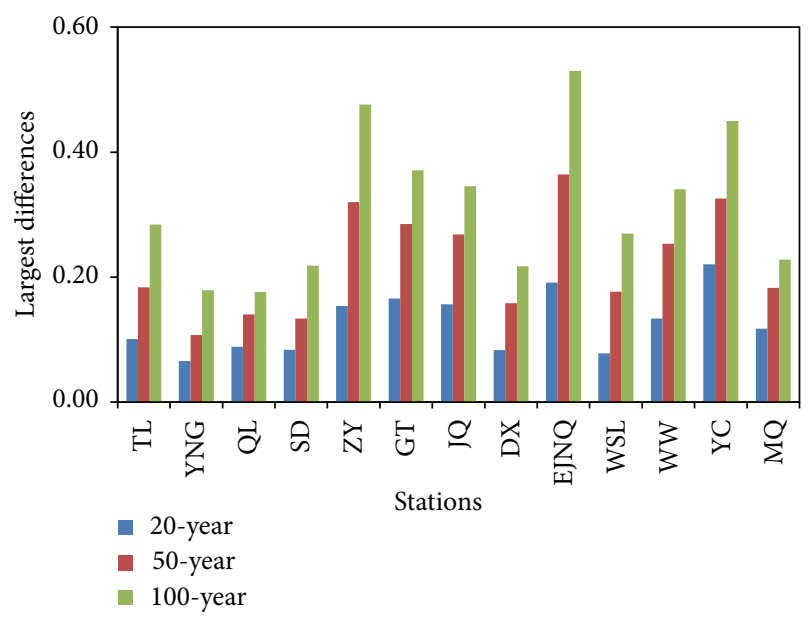

FIGURE 7: The largest difference in the estimated annual maximum precipitation derived from the alternative models and the best fitted ones on different return periods.

the set of hollow black circle denotes the empirical return period for the observed annual MP during the past 51 years. In this figure, two points are worth highlighting; one is that the estimated theoretical return levels from the top three distributions (GEV, Loglogistic, and Gumbel) most closely match the empirical ones in comparison, although all six distributions show good performance in the fitting based on $\mathrm{K}-\mathrm{S}$ test. Another point is that the estimated theoretical return levels differ depending on the distribution model used.

Since the estimations of return levels are different depending on the model we select, we try to discuss the degree of such difference caused by models. Suppose that $\widehat{x}_{0}$ means the return levels derived from the best fitted model which with the smallest statistic $D$ in K-S test and $\widehat{x}_{i}(i=$ $1,2, \ldots, 5)$ means those values from the other fitted models which are inferior to the most fitted model from the perspective of K-S test results. Then the difference is defined as $\alpha=$ $\left(\left(\widehat{x}_{i}-\widehat{x}_{0}\right) / \widehat{x}_{0}\right) \times 100 \%$. The smaller the value of $\alpha$, the smaller the difference resulting from the alternative model and the best fitted model. For the above specific example, compared with the best fitted model (GEV), Burr and Weibull show the larger deviations with differing by $8-10 \%, 16-18 \%$, and $20-$ $28 \%$ at 20 -year, 50 -year, and 100 -year return periods, respectively.

Figure 7 shows the greatest differences (largest $\alpha$ ) between the alternative models and the best fitted one on different return periods for all stations. Totally speaking, the largest difference among different stations displays as high as $22 \%, 36 \%$, and $53 \%$ on 20 -year, 50 -year, and 100 -year return periods, respectively, and the smallest difference among different stations also reaches as high as $6 \%, 11 \%$, and $18 \%$ on the above three return periods. It is quite comparable with the findings of Esteves [28] with differing by more than $40 \%$ depending on the distribution model used in the extreme precipitation estimates for long return periods. From the point of spatial scales, the differences in estimating return levels for the upper reaches (TL, YNG, QL, and WSL) present 
lower than those for the middle (SD, ZY, GT, JQ, WW, and YC) and lower reaches (DX, EJNQ, and MQ). Specifically, on average, it increases from $8 \%$ to $15 \%$ and $13 \%$ on 20 -year return period (in blue column) from the upper to the middle and lower reaches, increases from $15 \%$ to $16 \%$ and $23 \%$ on 50 -year return period (in red column), and increases from $22 \%$ to $23 \%$ and $33 \%$ on 100 -year return period (in green column). It is also clear that the longer the return periods, the greater the differences generated, which fairly corresponds to the features of extrapolations based on extreme value theory. Analysis for seasonal MP is omitted here due to the similar conclusions derived and limited space of the paper.

\section{Conclusions}

This study mainly investigates the performance of eight commonly used probability distributions for annual and seasonal maximum precipitation over two river basins in China and estimates the return levels on various return periods, with the purpose of both providing recommendation in the selection of suitable distribution for frequency analysis of precipitation extremes and better understanding the characteristics of extreme precipitation over the study areas. Daily precipitation records from 13 meteorological stations over two inland river basins are used. The goodness-of-fit is evaluated by comparing the calculated statistics $D$ in K-S test, together with such graphical diagnostics as probability difference graph, P-P plot, and cdf plot.

Results show that GEV, Burr, and Weibull distributions are proved to be the most suitable extreme value techniques in turn for both annual and seasonal MP over the two river basins. The worst performances are found for Exponential and Pareto 2 distributions even without passing the significance test for most MP of most stations. This leads to the recommendation that GEV, Burr, and Weibull distributions are preferable as means to model annual and seasonal maximum precipitation events for the dry inland river basins. We also note that most distributions selected here are relatively simple with only one shape parameter controlling their asymptotic behavior. Those distributions with more than one shape parameter may fit the tails of the data better; for example, Suhaila and Jemain [20] found that the mixed Gamma, mixed Weibull, and mixed Exponential showed better performances than the single distributions in modelling precipitation amounts in Peninsular Malaysia.

The estimated return levels based on the optimum models show that, for annual MP, they are higher for both upper and middle reaches than those for the lower reaches at shorter return period (20-year), while at moderate and longer return periods (50-year and 100-year), the estimates for the middle reaches show the highest. Estimates of Summer MP approximate to those of annual MP both in the quantity and in the spatial distributions. Estimates of Autumn MP show a little higher value than those of Spring MP, while the tendencies of spatial distributions present the same. As for Winter MP, very slight precipitation is estimated owing to the dry winter in the study areas. Another finding is that there are large differences among the estimated return levels derived from different distributions on various return periods. It differs by $22 \%, 36 \%$, and $53 \%$ at 20 -year, 50 -year, and 100 year return periods for annual maximum precipitation over two basins on average. It indicates that there are limitations to consider only one distribution model in extreme precipitation analysis, and cautions should be given to the protection of flood defense or infrastructure which are designed based on the estimated return levels.

\section{Conflict of Interests}

The authors declare that there is no conflict of interests regarding the publication of this paper.

\section{Acknowledgments}

The authors thank anonymous reviewer(s) for valuable comments and suggestions to improve the quality of this paper. This study is supported by Beijing Higher Education Yong Elite Teacher Project (YETP0654), the Fundamental Research Funds for the Central Universities (no. 2652012082), and NSFC (no. 41101038).

\section{References}

[1] M. Gemmer, T. Fischer, T. Jiang, B. Su, and L. L. Liu, "Trends in precipitation extremes in the Zhujiang River Basin, South China," Journal of Climate, vol. 24, no. 3, pp. 750-761, 2011.

[2] J. V. Revadekar, S. K. Patwardhan, and K. Rupa Kumar, "Characteristic features of India in the warming scenarios," Advances in Meteorology, vol. 2011, Article ID 138425, 11 pages, 2011.

[3] S. M. Papalexiou, D. Koutsoyiannis, and C. Makropoulos, "How extreme is extreme? An assessment of daily rainfall distribution tails," Hydrology and Earth System Sciences, vol. 17, no. 2, pp. 851862, 2013.

[4] H. Deng, Y. Chen, X. Shi et al., "Dynamics of temperature and precipitation extremes and their spatial variation in the arid region of Northwest China," Atmospheric Research, vol. 138, pp. 346-355, 2014

[5] Y. D. Chen, Q. Zhang, M. Xiao, V. P. Singh, Y. Leung, and L. Jiang, "Precipitation extremes in the Yangtze River Basin, China: regional frequency and spatial-temporal patterns," Theoretical and Applied Climatology, vol. 116, no. 3-4, pp. 447-461, 2014.

[6] E. Shamir, K. P. Georgakakos, and M. J. Murphy, "Frequency analysis of the 7-8 December 2010 extreme precipitation in the Panama Canal Watershed," Journal of Hydrology, vol. 480, pp. 136-148, 2013.

[7] J. Feng, D. Yang, C. Li, Y. Gao, and J. Liu, "Regional frequency analysis of extreme precipitation after drought events in the Heihe River Basin, Northwest China," Journal of Hydrologic Engineering, vol. 19, no. 6, pp. 1101-1112, 2014.

[8] H. Du, J. Xia, S. Zeng, D. She, and J. Liu, "Variations and statistical probability characteristic analysis of extreme precipitation events under climate change in Haihe River Basin, China," Hydrological Processes, vol. 28, no. 3, pp. 913-925, 2014.

[9] S. G. Coles, An Introduction to Statistical Modeling of Extreme Values, Springer, Berlin, Germany, 2001.

[10] Z. Li, F. Brissette, and J. Chen, "Assessing the applicability of six precipitation probability distribution models on the Loess Plateau of China," International Journal of Climatology, vol. 34, no. 2, pp. 462-471, 2014. 
[11] Z. Li, F. Brissette, and J. Chen, "Finding the most appropriate precipitation probability distribution for stochastic weather generation and hydrological modelling in Nordic watersheds," Hydrological Processes, vol. 27, no. 25, pp. 3718-3729, 2013.

[12] V. Rahmani, S. L. Hutchinson, J. M. S. Hutchinson, and A. Anandhi, "Extreme daily rainfall event distribution patterns in Kansas," Journal of Hydrologic Engineering, vol. 19, no. 4, pp. 707-716, 2014.

[13] J. Xia, H. Du, S. Zeng et al., "Temporal and spatial variations and statistical models of extreme runoff in Huaihe River Basin during 1956-2010," Journal of Geographical Sciences, vol. 22, no. 6, pp. 1045-1060, 2012.

[14] L. Benyahya, P. Gachon, A. St-Hilaire, and R. Laprise, "Frequency analysis of seasonal Extreme precipitation in southern Quebec (Canada): an evaluation of regional climate model simulation with respect to two gridded datasets," Hydrology Research, vol. 45, no. 1, pp. 115-133, 2014.

[15] A. S. Rahman, A. Rahman, M. A. Zaman, K. Haddad, A. Ahsan, and M. Imteaz, "A study on selection of probability distributions for at-site flood frequency analysis in Australia," Natural Hazards, vol. 69, no. 3, pp. 1803-1813, 2013.

[16] J. L. Salinas, A. Castellarin, S. Kohnová, and T. R. Kjeldsen, "On the quest for a pan-European flood frequency distribution: effect of scale and climate," Hydrology and Earth System Sciences Discussions, vol. 10, no. 5, pp. 6321-6358, 2013.

[17] D. S. Wilks, "Interannual variability and extreme-value characteristics of several stochastic daily precipitation models," Agricultural and Forest Meteorology, vol. 93, no. 3, pp. 153-169, 1999.

[18] T. Fischer, B. Su, Y. Luo, and T. Scholten, "Probability distribution of precipitation extremes for weather index-based insurance in the Zhujiang River Basin, South China," Journal of Hydrometeorology, vol. 13, no. 3, pp. 1023-1037, 2012.

[19] H. Wan, X. Zhang, and E. M. Barrow, "Stochastic modelling of daily precipitation for Canada," Atmosphere-Ocean, vol. 43, no. 1, pp. 23-32, 2005.

[20] J. Suhaila and A. A. Jemain, "Fitting daily rainfall amount in Peninsular Malaysia using several types of exponential distributions," Journal of Applied Sciences Research, vol. 27, no. 10, pp. 1027-1036, 2007.

[21] S. J. Asl, A. M. Khorshiddoust, Y. Dinpashoh, and F. Sarafrouzeh, "Frequency analysis of climate extreme events in Zanjan, Iran," Stochastic Environmental Research and Risk Assessment, vol. 27, no. 7, pp. 1637-1650, 2013.

[22] S. D. Grimshaw, "Computing maximum likelihood estimates for the generalized Pareto distribution," Technometrics, vol. 35, no. 2, pp. 185-191, 1993.

[23] R. Deidda and M. Puliga, "Performances of some parameter estimators of the generalized Pareto distribution over roundedoff samples," Physics and Chemistry of the Earth, vol. 34, no. 1012, pp. 626-634, 2009.

[24] S. G. Coles and M. J. Dixon, "Likelihood-Based inference for extreme value models," Extremes, vol. 2, no. 1, pp. 5-23, 1999.

[25] I. M. Chakravarti, R. G. Laha, and J. Roy, Handbook of Methods of Applied Statistics, vol. 1, John Wiley \& Sons, 1967.

[26] J. Grieser, T. Staeger, and C.-D. Schonwiese, "Estimates and uncertainties of return periods of extreme daily precipitation in Germany," Meteorologische Zeitschrift, vol. 16, no. 5, pp. 553564, 2007.

[27] J. Kyselý, L. Gaál, J. Picek, and M. Schindler, "Return periods of the August 2010 heavy precipitation in northern Bohemia
(Czech Republic) in the present climate and under climate change," Journal of Water and Climate Change, vol. 4, no. 3, pp. 265-286, 2013.

[28] L. S. Esteves, "Consequences to flood management of using different probability distributions to estimate extreme rainfall," Journal of Environmental Management, vol. 115, pp. 98-105, 2013.

[29] Q. X. Shao, H. Wong, J. Xia, and W.-C. Ip, "Models for extremes using the extended three-parameter Burr XII system with application to flood frequency analysis," Hydrological Sciences Journal, vol. 49, no. 4, pp. 685-702, 2004.

[30] D. She, J. Xia, J. Song, H. Du, J. Chen, and L. Wan, "Spatiotemporal variation and statistical characteristic of extreme dry spell in Yellow River Basin, China," Theoretical and Applied Climatology, vol. 112, no. 1-2, pp. 201-213, 2013.

[31] C. Scarrott and A. MacDonald, "A review of extreme value threshold estimation and uncertainty quantification," REVSTAT Statistical Journal, vol. 10, no. 1, pp. 33-60, 2012. 

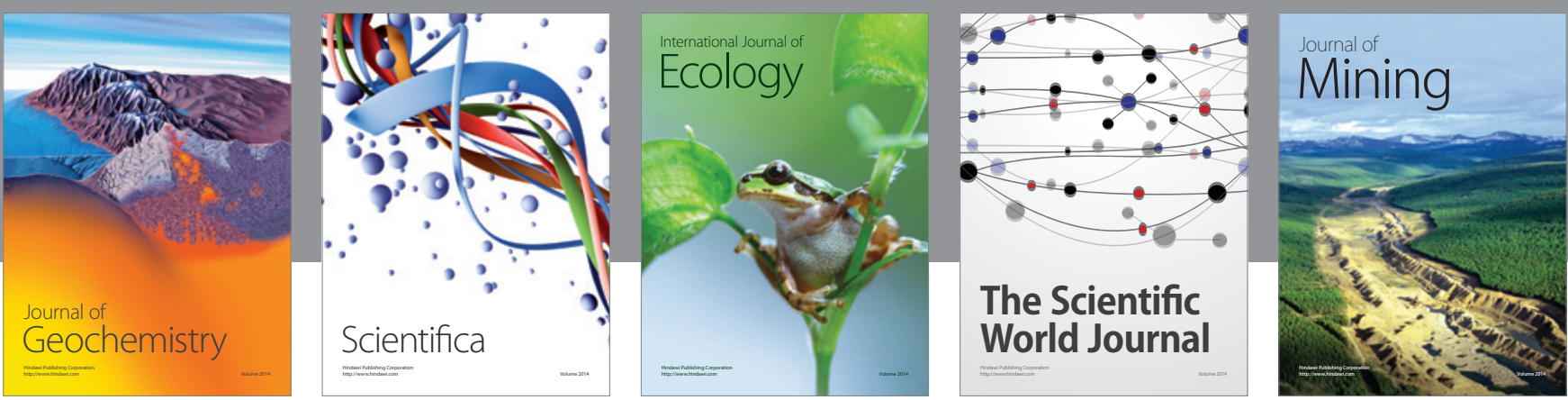

The Scientific World Journal
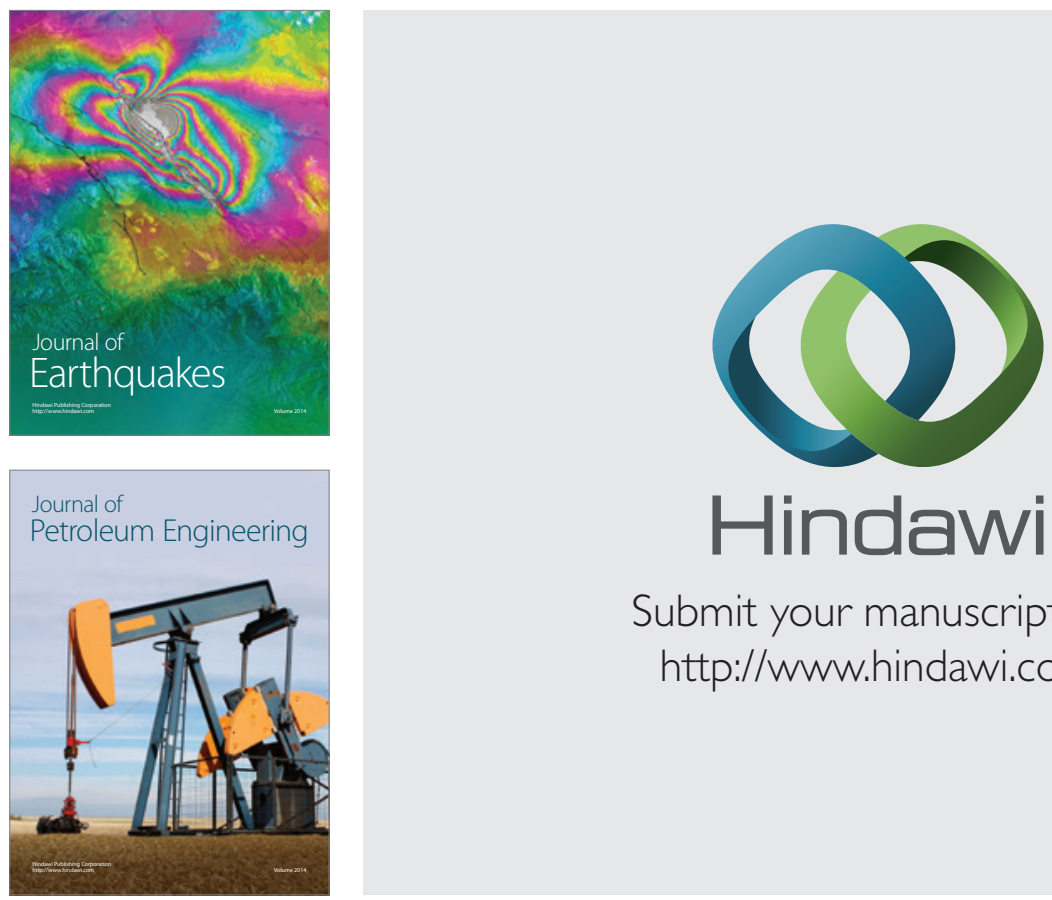

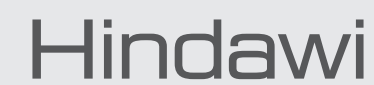

Submit your manuscripts at

http://www.hindawi.com
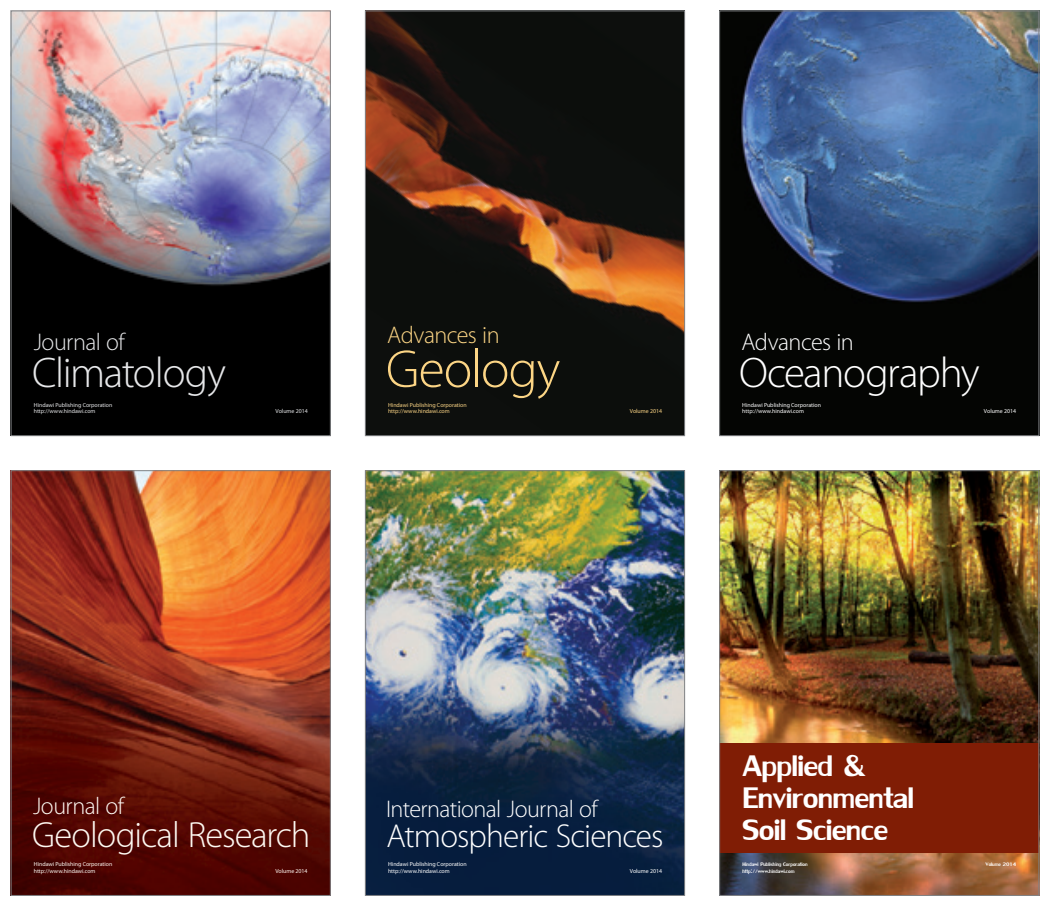
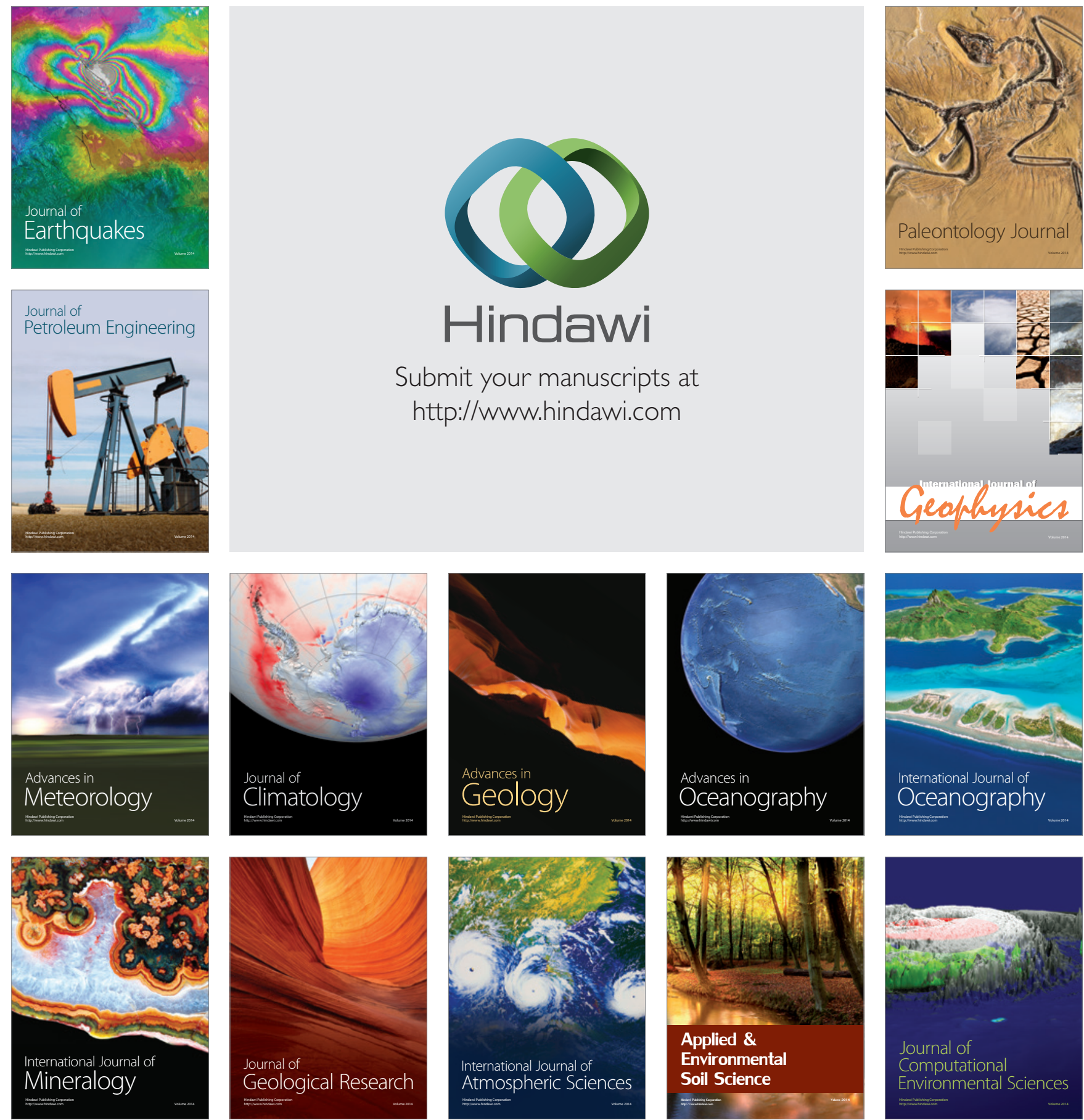